\title{
Decision making in reinsurance with induced OWA operators and
}

\section{Minkowski distances}

\author{
Montserrat Casanovas ${ }^{1}$, Agustín Torres-Martínez ${ }^{1}$ José M. Merigó $^{2 *}$ \\ ${ }^{I}$ Department of Business Administration, University of Barcelona, \\ Av. Diagonal 690, 08034 Barcelona, Spain \\ ${ }^{2}$ Department of Management Control and Information Systems, University of Chile, \\ Av. Diagonal Paraguay 257, 8330015 Santiago, Chile
}

\begin{abstract}
The decision to choose a reinsurance program has many complexities since it is difficult to get simultaneously high levels in different optimal criteria including maximum gain, minimum variance and probability of ruin. This article suggests a new method in which through membership functions we can measure the distance of each alternative to an optimal result and aggregate it by using different types of aggregations. In this paper, particular attention is given to the induced Minkowski ordered weighted averaging distance operator and the induced Minkowski probabilistic ordered weighted averaging distance operator. The main advantage of these operators is that they include a wide range of special cases. Thus, they can adapt efficiently to the specific needs of the calculation processes. By doing so, the reinsurance system can make better decisions by using different scenarios in the uncertain environment considered.
\end{abstract}

Keywords: Reinsurance, Minkowski distance, OWA operators, Fuzzy logic.

\footnotetext{
* Corresponding author. Tel: +34 934021962 ; Fax: +34 934039882

E-mail address: jmerigo@fen.uchile.cl (J.M. Merigó).
} 


\section{Introduction}

Reinsurance is an instrument used by insurance companies to align risks with their financial capacity by transferring all or part of the risk assumed to reinsurer to not jeopardize the solvency of the insurer. But otherwise this level of reinsurance reduces the profits of the company by sharing part of its income from premiums. As in other problems of the insurance sector in the case of reinsurance, the statistical tools have proven very effective in their management. However, when deciding on a type of reinsurance the insurance company faces decisions involving internal and external factors that are determined by the general environment such as the expected economic situation and by the specific environment as legal regulations of the sector.

The important role of reinsurance in the insurance sector is beyond dispute. So the decisions in insurance companies will have a direct and highly relevant influence on their organizational goals. In this regard it is important to highlight some of the basic reasons for hiring reinsurance. First, for spreading the risk of a possible loss, either partially or completely. Next, for taking risks for an amount exceeding what the company can cope with their own resources. Finally, to increase the volume of business or facilitating the emergence of new insurers.

These reasons lead to insurers to be primarily based on the criteria of profitability and solvency to decide on a particular reinsurance, being important to note that as well as quantitative information, there are other subjective criteria for decisionmaking. That is why in this article a method is proposed using tools such as fuzzy logic, fuzzy numbers and OWA operators (Yager, 1988; Yager et. al. 2011) with Minkowski distances to evaluate alternative reinsurance systems. This evaluation is based on the use of reinsurance optimization criteria (maximum gain, minimum 
variance and minimum probability of ruin), in which it is very difficult for an alternative to be better than the others in all criteria at once. Therefore, from an optimal level of acceptance defined by experts, the alternatives are compared using Hamming and Euclidean distances. They are generalized with the Minkowski distance and are added with OWA operators, through the IMOWAD operator (Merigó \& Casanovas, 2011a, $2011 b, 2011 c)$ to make decisions involving the optimistic or pessimistic attitude of the decision maker.

Note that in the literature there are a variety of new operators with distances among which are the OWA distance (Merigó \& Gil-Lafuente, 2010, Xu \& Chen, 2008), continuous distances (Zhou et al 2013), distances with Choquet integral (Bolton et al., 2008), the probabilistic weighted averaging distance (PWAD) (Merigó, 2013), the immediate probabilities (Merigó \& Gil-Lafuente, 2012), the probabilistic OWA distance (Merigó et al. 2013) and moving distances (Merigó \& Yager, 2013). Other models have focused on the use of imprecise information with distances including intervals numbers (Zeng, 2013a) and fuzzy numbers (Su et al 2013; Xu, 2013; Zeng, 2013b). Moreover, other related tools have been developed using norms such as the adequacy coefficient (Merigó \& Gil-Lafuente, 2010, 2013).

The paper is organized as follows. Section 2 reviews some basic preliminaries on reinsurance, OWA operators and distance measures. Section 3 analyzes some modern distance aggregation operators and Section 4 presents an application of these aggregation operators in a reinsurance problem. Section 5 summarizes the main results and conclusions of the paper. 


\section{Preliminaries}

This section consists of two parts. The first one explains the main classes of reinsurance and criteria for choosing an optimal reinsurance and the second part reviews the concepts of OWA operators and distance measures.

\subsection{Reinsurance}

The reinsurance is a type of contract in which an insurer assumes the risk fully or partially covered by another insurer and the transferor may take on more risk by ensuring the solvency of the company. The three most common types of reinsurance are:

1) Quota Share Reinsurance: is a type of proportional reinsurance where the insurer transfers a fixed proportion of the amount of losses to the reinsurer. It is defined as:

$$
X_{r e a_{i}}=\operatorname{Min}\left[(l-k) X_{i}, L_{1}\right] \forall X_{i} i=1,2, \ldots, n,
$$

where $X_{r e a_{i}}$ is the amount that the reinsurer pays for each claim, $k$ is the proportion of the individual amount corresponding to the transferor and $L_{1}$ is the maximum limit of liability.

2) Excess of Loss Reinsurance Risk: is a type of non-proportional contract called deductible, in which the reinsurer agrees to assume claims above this amount until the limit of liability. It is defined as: 


$$
X_{\text {rea }_{i}}= \begin{cases}0 & X_{i} \leq M \\ \operatorname{Min}\left(X_{i}, L_{2}\right) & X_{i}>M\end{cases}
$$

where $X_{r e a_{i}}$ is the amount that the reinsurer pays for each claim, $M$ is deductible assuming the transferor and $L_{2}$ is the maximum limit of liability.

3) Stop Loss Reinsurance: In this type of contract called priority, where the reinsurer covers the amount of losses from certain amount of responsibility to the maximum. It is defined as:

$$
X_{\text {rea }}= \begin{cases}0 & X_{i} \leq d \\ \operatorname{Min}\left(X_{i}, L_{2}\right)-d & X_{i}>d\end{cases}
$$

where $X_{\text {rea }}$ is the amount that the reinsurer pays for each claim, $d$ is the aggregate amount of claims assumed by the transferor and $L_{2}$ is the maximum limit of liability.

\subsection{Criteria for optimal reinsurance}

The reinsurance decision is a fairly complex process, because it must reconcile conflicting aims, being very important the level of risk you are willing to take of the insurer depending on the circumstances and environment. The most important criteria in this type of decision (Mondragón, 2009) are presented below: 
1) Maximum Gain: Earnings for the direct insurer are obtained through the following expression:

$$
G=P-X-P R+X_{\text {rea }},
$$

where $P$ is the risk premium charged by the insurer regardless of the type of reinsurance, $X$ is the aggregate amount of claims, $P R$ is the reinsurance premium and $X_{\text {rea }}$ is the aggregate amount of claims recovered.

Keep in mind that:

- $\quad X$ and $X_{\text {rea }}$ are random variables.

- $\quad P R$ and $X_{\text {rea }}$ depend on the chosen reinsurance.

2) Minimum Variance: In this approach the optimal reinsurance is one in which the aggregate amount of retained claims $\left(X-X_{\text {rea }}\right)$ is minimal. It is important to consider the values of $P R$ on this criterion, since the aim is to get the minimum variance at the lowest cost.

An extension appropriate in this case may be the coefficient of variation of the portfolio that corresponds to the ratio between the standard deviation and the arithmetic mean.

3) Low Probability of Ruin: The solvency of the insurer is a very important criterion for reinsurance decisions, this being assessed through the ruin probability. In this case an insurer will ruin when:

$$
G+C I<0
$$


where $G$ is the gain and $C I$ is the capital of the insurer at the beginning of the period, where the probability of ruin for initial capital is given by:

$$
\psi(C I)=P[G+C I<0] .
$$

Importantly, $G$ depends on the chosen reinsurance and thus influences the probability of ruin.

As can be seen, it is very complex that reinsurance programs meet all three criteria, because if for example you have a low variance, it makes sense that earnings are affected by having a higher premium and reinsurance vice versa. Thus reinsurance decisions influence the importance that is given to each of the criteria compared to others and the risk you are willing to take and this will depend on the optimistic or pessimistic attitude of decision maker, being very useful in these cases using OWA aggregation operators.

\subsection{Fuzzy logic and aggregation operators}

\subsubsection{Fuzzy membership functions}

They were introduced by Zadeh (1965) to define the fuzzy sets, taking an element as an argument and assigning a value between 0 and 1, which relates the level of membership of the element to that set. Some of the most commonly used membership functions are the following ones: 


$$
\begin{gathered}
\Gamma\left(U_{A}(x)\right)=\left\{\begin{array}{lr}
0 & \begin{array}{r}
x \\
\left(\frac{x-a}{b-a}\right)
\end{array} \\
1 & a<x<b
\end{array}\right. \\
\mathrm{L}\left(U_{A}(x)\right)=\left\{\begin{array}{lr}
1 & x \geq b \\
\left(\frac{b-x}{b-a}\right) & a<x<b \\
0 & x \geq b
\end{array}\right. \\
\wedge\left(U_{A}(x)\right)=\left\{\begin{array}{lr}
1 & x \leq a \\
\left(\frac{x-a}{b-a}\right) & a<x \leq b \\
\left(\frac{c-x}{c-b}\right) & b<x<c \\
0 & x \geq c
\end{array} .\right.
\end{gathered}
$$

\subsubsection{The OWA operator}

The OWA operator provides a parameterized family of aggregation operators (Beliakov et. al., 2007; Grabisch et. al., 2011) between the minimum and maximum. In this way, information can be added depending on the degree of optimism or pessimism that the decision maker wants to take in the decision process (Belles et al. 2013; Figueira et. al., 2005; Zavadskas and Turskis, 2011).

Definition 1: An OWA operator is defined as a mapping of dimension $n, F: \Re^{n} \rightarrow \Re$, that has an associated weighting vector $W$ of dimension $n, W=\left[w_{1}, w_{2}, \ldots, w_{n}\right]^{T}$, that meets the following conditions:

- $\quad w_{j} \in[0,1]$

$-\quad \sum_{j=1}^{n} w_{j}=1$

$-f\left(a_{1}, a_{2}, \ldots, a_{n}\right)=\sum_{j=1}^{n} w_{j} \cdot b_{j}$ 
The essence of the OWA (Yager, 1988; 1993; 1998) is the rearrangement of the elements or arguments, causing the aggregation in $a_{j}$ are not associated with a weighting $w_{j}$, but the $w_{j}$ will be associated with the position in the order for aggregation.

Under this context, it is also possible to define the ascending OWA operator as follows.

Definition 2: An Ascending OWA (AOWA) operator is defined as a mapping of dimension $n, F: \mathfrak{R}^{n} \rightarrow \mathfrak{R}$, that has an associated weighting vector $W$ of dimension $n$, $W=\left[w_{1}, w_{2}, \ldots, w_{n}\right]^{T}$, that meets the following conditions:

- $\quad w_{j} \in[0,1]$

$-\quad \sum_{j=1}^{n} w_{j}=1$

- $f\left(a_{1}, a_{2}, \ldots, a_{n}\right)=\sum_{j=1}^{n} w_{j} \cdot b_{j}$

where $b_{j}$ is the $j$ th smallest of the $a_{i}$, such that, $b_{1} \leq b_{2} \leq \cdots \leq b_{n}$, which thus differ from the OWA where $b_{1} \geq b_{2} \geq \cdots \geq b_{n}$.

The difference between the OWA operator (Descending OWA) and the AOWA is the way it manages the arguments, descending in the first and ascending in the second, respectively, and depends on the optimistic or pessimistic attitudes of the decision maker. Note that these two operators are related by using $w_{j}=w^{*}{ }_{n-j+1}$, where $w_{j}$ is the $j$ th weight of the AOWA and $w^{*}{ }_{n-j+1}$ the $j$ th weight of the DOWA operator. 


\subsubsection{Distance measures}

The notion of distance is used to measure the difference or calculate the degree of distance between two elements or sets (Gil-Aluja, 1999; Kaufmann, 1975). The distances most commonly used are the Hamming distance, the Euclidean distance and the Minkowski distance. It can be considered a distance measure, provided that the following properties are satisfied:

1. No negativity: $D\left(A_{1}, A_{2}\right) \geq 0$.

2. Conmutativity: $D\left(A_{1}, A_{2}\right)=D\left(A_{2}, A_{1}\right)$.

3. Reflexivity: $D\left(A_{1}, A_{1}\right)=0$.

4. Triangle Inequality: $D\left(A_{1}, A_{2}\right)+D\left(A_{2}, A_{3}\right) \geq D\left(A_{1}, A_{3}\right)$.

The Hamming distance can be formulated in the following two ways (Hamming, 1950):

Definition 3 (Discrete Scope): Let $E$ be a finite referential and $A, B \subset E$, so that the Hamming distance is defined as:

$$
d(A, B)=\sum_{i=1}^{n}\left|u_{\underline{A}}\left(x_{i}\right)-u_{\underline{B}}\left(x_{i}\right)\right| \text {. }
$$

with $x \in E \forall i=1,2, \ldots, n ; u_{A}\left(x_{i}\right), u_{B}\left(x_{i}\right) \epsilon[0,1]$.

Definition 4 (Continuous Scope): Assuming that the reference $E$ is the set of real numbers $(E=R)$, the Hamming distance $\forall x \in\left[x_{1}, x_{2}\right]$ is defined as: 


$$
d(A, B)=\int_{x_{1}}^{x_{2}}\left|u_{\underline{A}}\left(x_{i}\right)-u_{\underline{B}}\left(x_{i}\right)\right| d x .
$$

The Euclidean distance can be formulated in the following ways:

Definition 6 (Discrete Scope): Let $E$ a finite referential and $A, B \subset E$, so that the Euclidean distance is defined as:

$$
e(A, B)=\sqrt{\sum_{i=1}^{n}\left(u_{\underline{A}}\left(x_{i}\right)-u_{\underline{B}}\left(x_{i}\right)\right)^{2}},
$$

with $x \in E \forall i=1,2, \ldots, n ; u_{\underline{A}}\left(x_{i}\right), u_{\underline{B}}\left(x_{i}\right) \epsilon[0,1]$.

Definition 7 (Continuous Scope): Assuming that the reference $E$ is the set of real numbers $(E=R)$, the Euclidean distance $\forall x \in\left[x_{1}, x_{2}\right]$ is defined as:

$$
e(A, B)=\sqrt{\int_{x_{1}}^{x_{2}}\left(u_{\underline{A}}\left(x_{i}\right)-u_{\underline{B}}\left(x_{i}\right)\right)^{2} d x} .
$$

The Minkowski distance is a generalization of a wide range of distances that includes the Euclidean and Hamming distances and is formulated as follows:

Definition 8 (Discrete Scope): Let $E$ a finite referential and $A, B \subset E$, so that the Minkowski distance is defined as:

$$
e(A, B)=\left(\sum_{i=1}^{n}\left|u_{\underline{A}}\left(x_{i}\right)-u_{\underline{B}}\left(x_{i}\right)\right|^{\lambda}\right)^{1 / \lambda},
$$


with $x \in E \forall i=1,2, \ldots, n ; u_{\underline{A}}\left(x_{i}\right), u_{\underline{B}}\left(x_{i}\right) \epsilon[0,1]$ and $\lambda \in N-\{0\}$.

Definition 7 (Continuous Scope): Assuming that the reference $E$ is the set of real numbers $(E=R)$, the Minkowski distance $\forall x \in\left[x_{1}, x_{2}\right]$ is defined as:

$$
r(A, B)=\left(\int_{x_{1}}^{x_{2}}\left|u_{\underline{A}}\left(x_{i}\right)-u_{\underline{B}}\left(x_{i}\right)\right|^{\lambda} d x\right)^{1 / \lambda},
$$

with $\lambda \in N-\{0\}$.

In the Minkowski distance, both in the discrete and continuous scopes, we have:

- If $\lambda=1$, it becomes the Hamming distance.

- If $\lambda=2$, it becomes the Euclidean distance.

The normalized Minkowski distance corresponds to a measure that uses arithmetic means in the aggregation process and generalizes a wide range of distances such as the normalized Hamming distance and the normalized Euclidean distance. This distance may be formulated to two sets A and B as follows:

Definition 10: A normalized Minkowski distance of dimension $n$, is a mapping $d_{m}: R^{n} x R^{n} \rightarrow R$, such that:

$$
d_{m}(A, B)=\frac{1}{n}\left(\sum_{i=1}^{n}\left|a_{i}-b_{i}\right|^{\lambda}\right)^{1 / \lambda},
$$


where $a_{i}$ and $b_{i}$ are the arguments of the sets $\mathrm{A}$ and $\mathrm{B}$ and $\lambda$ is a parameter such that $\lambda \in$ $(-\infty, \infty)$

The weighted Minkowski distance is applied in cases where it is preferred to give different weights to the individual distance. It is defined as follows:

Definition 11: A weighted Minkowski distance of dimension $n$ is a mapping $d_{m}: R^{n} x R^{n} \rightarrow R$, which has an associated weighting vector $W$ of dimension $n$ such that $\sum_{i=1}^{n} w_{i}=1$ and $w_{i} \in[0,1]$. So that:

$$
d_{m}(A, B)=\left(\sum_{i=1}^{n} w_{i}\left|a_{i}-b_{i}\right|^{\lambda}\right)^{1 / \lambda},
$$

where $a_{i}$ and $b_{i}$ are the arguments of the sets $\mathrm{A}$ and $\mathrm{B}$ and $\lambda$ is a parameter such that $\lambda \in$ $(-\infty, \infty)$

If different values for the parameter $\lambda$ are formulated, we can obtain a wide range of special cases. For example:

- If $\lambda=1$, it becomes the weighted Hamming distance.

- If $\lambda=2$, it becomes the weighted Euclidean distance. 


\section{OWA operators and Minkowski distances}

\subsection{Introduction}

Next, let us study some Minkowski distances that use different types of OWA operators. The Minkowski OWA distance (MOWAD) operator (Merigó and Casanovas, 2011a) represents an extension of the normalized Minkowski distance using OWA operators, with the difference that the rearrangement of the distances of the arguments is in accordance with their values, so that it takes into account the attitudinal character of the decision maker. It is defined as follows:

Definition 12: The MOWAD operator of dimension $n$, is a mapping: MOWAD: $R^{n} x R^{n} \rightarrow R$, which has an associated weighting vector $W$, of dimension $n$ such that $\sum_{i=1}^{n} w_{i}=1$ and $w_{i} \in[0,1]$. So that:

$$
\operatorname{MOWAD}\left(d_{1}, d_{2}, \ldots, d_{n}\right)=\left(\sum_{i=1}^{n} w_{i} D_{i}^{\lambda}\right)^{1 / \lambda}
$$

where $D_{i}$ is the $j$ th largest of the $d_{i}, d_{i}$ is the individual distance between A and B, such that $d_{i}=\left|a_{i}-b_{i}\right|$ and $\lambda$ is a parameter such that $\lambda \in(-\infty, \infty)$.

If different values for the parameter $\lambda$ are formulated, you can have a wide range of special cases. For example:

- If $\lambda=1$, it becomes the Hamming OWA distance operator. 
- If $\lambda=2$, it becomes the Euclidean OWA distance operator.

Another interesting extension is the induced MOWAD (IMOWAD) operator. The IMOWAD (or IGOWAD) operator (Merigó \& Casanovas, 2011a) is an aggregation operator, in which the ordering of the arguments does not depend on their individual characteristics, but rather on the induced sorting variables and is defined as follows:

Definition 13: The IMOWAD operator of dimensionn, is a mapping IMOWAD: $R^{n} x R^{n} \rightarrow R$, which has an associated weighting vector $W$ of dimension $n$ such that $\sum_{i=1}^{n} w_{i}=1$ and $w_{i} \in[0,1]$. So that:

$$
\operatorname{IMOWAD}\left(\left\langle u_{1}, a_{1}, b_{1}\right\rangle,\left\langle u_{2}, a_{2}, b_{2}\right\rangle, \ldots,\left\langle u_{n}, a_{n}, b_{n}\right\rangle\right)=\left(\sum_{i=1}^{n} w_{i} D_{i}^{\lambda}\right)^{1 / \lambda}
$$

where $D_{i}$ is the $d_{i}=\left|a_{i}-b_{i}\right|$, value of the triplet IMOWAD $\left\langle u_{i}, a_{i}, b_{i}\right\rangle$ with the $j$-th largest $u_{i}, u_{i}$ is the induced ordering variable, $\left|a_{i}-b_{i}\right|$ is the argument variable that is represented by individual distances and $\lambda$ is a parameter such that $\lambda \in(-\infty, \infty)$.

If different values for the parameter $\lambda$ are formulated, you can have a wide range of special cases. For example:

- If $\lambda=1$, it becomes the IHOWAD operator (Merigó \& Casanovas, 2011b).

- If $\lambda=2$, it becomes the IEOWAD operator (Merigó \& Casanovas, 2011c). 


\subsection{The Minkowski probabilistic OWA distance}

The Minkowski probabilistic ordered weighted averaging distance (MPOWAD) operator is a distance aggregation operator that provides a unified framework between the probability and the OWA operator. It also uses generalized means providing a general formulation that includes a wide range of particular cases including the probabilistic OWA distance (POWAD) (Merigó et al. 2013) and the Euclidean probabilistic ordered weighted averaging distance (EPOWAD). Note that the probability can also be seen as the weighted average. For two sets $X=\left\{x_{1}, x_{2}, \ldots, x_{n}\right\}$ and $Y=\left\{y_{1}, y_{2}, \ldots, y_{n}\right\}$, it can be defined as follows.

Definition 14. A MPOWAD operator is a mapping MPOWAD: $R^{n} \times R^{n} \rightarrow R$ of dimension $n$, if it has an associated weighting vector $W$, with $\sum_{j=1}^{n} w_{j}=1$ and $w_{j} \in[0$, 1] and a weighting vector $V$ that affects the probability, with $\sum_{i=1}^{n} v_{i}=1$ and $v_{i} \in[0,1]$, such that:

$$
\operatorname{MPOWAD}\left(\left\langle x_{1}, y_{1}\right\rangle, \ldots,\left\langle x_{n}, y_{n}\right\rangle\right)=\beta\left(\sum_{j=1}^{n} w_{j} b_{j}^{\lambda}\right)^{1 / \lambda}+(1-\beta)\left(\sum_{i=1}^{n} v_{i}\left|x_{i}-y_{i}\right|^{\sigma}\right)^{1 / \sigma}
$$

where $b_{j}$ is the $j$ th smallest of the $\left|x_{i}-y_{i}\right|, x_{i}$ is the $i$ th argument of the set $X=\left\{x_{1}, \ldots\right.$, $\left.x_{n}\right\}, y_{i}$ is the $i$ th argument of the set $Y=\left\{y_{1}, \ldots, y_{n}\right\}, \beta \in[0,1]$ and $\lambda$ and $\sigma$ are parameters such that $\lambda, \sigma \in(-\infty, \infty)-\{0\}$. 
Note that it is possible to distinguish between ascending (AMPOWAD) and descending (DMPOWAD) orders by using $w_{j}=w^{*}{ }_{n-j+1}$, where $w_{j}$ is the $j$ th weight of the AMPOWAD and $w^{*}{ }_{n-j+1}$ the $j$ th weight of the DMPOWAD operator. The MPOWAD operator is bounded, idempotent and monotonic. It is also reflexive and non-negative. If $\lambda=\sigma=1$, the MPOWAD operator becomes the POWAD operator (Merigó et al. 2013):

$$
\operatorname{POWAD}\left(\left\langle x_{1}, y_{1}\right\rangle, \ldots,\left\langle x_{n}, y_{n}\right\rangle\right)=\beta\left(\sum_{j=1}^{n} w_{j} b_{j}\right)+(1-\beta)\left(\sum_{i=1}^{n} v_{i}\left|x_{i}-y_{i}\right|\right),
$$

And if $\lambda=\sigma=2$, the MPOWAD becomes the EPOWAD operator:

$$
\operatorname{EPOWAD}\left(\left\langle x_{1}, y_{1}\right\rangle, \ldots,\left\langle x_{n}, y_{n}\right\rangle\right)=\beta \sqrt{\left(\sum_{j=1}^{n} w_{j} b_{j}^{2}\right)}+(1-\beta) \sqrt{\left(\sum_{i=1}^{n} v_{i}\left|x_{i}-y_{i}\right|^{2}\right)}
$$

\subsection{The induced Minkowski probabilistic OWA distance}

The induced Minkowski probabilistic ordered weighted averaging distance (IMPOWAD) operator is a distance measure that uses a unified framework between the probability and the IOWA operator (Yager and Filev, 1999) in the normalization process of the Minkowski distance. Thus, the reordering of the individual distances is developed according to order-inducing variables that represent a complex reordering process of the individual distances. Moreover, it also uses generalized means. Therefore, it includes a wide range of distance aggregation operators including the induced POWAD (IPOWAD) operator and the induced Euclidean POWAD (IEPOWAD) operator. 
The first main advantage of this new approach is that it is able to deal with situations where we have some objective information about the possibility of occurrence of the different results. The second main advantage is the use of a parameterized family of aggregation operators between the minimum and the maximum. This is assessed with order-inducing variables in order to represent the attitudinal character of the decision maker in a complete way, considering a wide range of aspects such as the degree of optimism, psychological aspects and time pressure. For two sets $X=\left\{x_{1}, x_{2}, \ldots, x_{n}\right\}$ and $Y=\left\{y_{1}, y_{2}, \ldots, y_{n}\right\}$, it can be defined as follows.

Definition 15. An IMPOWAD operator is a mapping IMPOWAD: $R^{n} \times R^{n} \times R^{n} \rightarrow R$ of dimension $n$, if it has an associated weighting vector $W$, with $\sum_{j=1}^{n} w_{j}=1$ and $w_{j} \in[0$, 1] and a weighting vector $V$ that affects the probability, with $\sum_{i=1}^{n} v_{i}=1$ and $v_{i} \in[0,1]$, such that:

$$
\begin{aligned}
& \operatorname{IMPOWAD}\left(\left\langle u_{1}, x_{1}, y_{1}\right\rangle, \ldots,\left\langle u_{n}, x_{n}, y_{n}\right\rangle\right)= \\
& =\beta\left(\sum_{j=1}^{n} w_{j} b_{j}^{\lambda}\right)^{1 / \lambda}+(1-\beta)\left(\sum_{i=1}^{n} v_{i}\left|x_{i}-y_{i}\right|^{\sigma}\right)^{1 / \sigma},
\end{aligned}
$$

where $b_{j}$ is the $\left|x_{i}-y_{i}\right|$ value of the IGPOWAD triplet $\left\langle u_{i}, x_{i}, y_{\mathrm{i}}\right\rangle$ having the $j$ th largest $u_{i}$, $u_{i}$ is the order-inducing variable, $x_{i}$ is the $i$ th argument of the set $X=\left\{x_{1}, \ldots, x_{n}\right\}, y_{i}$ is the $i$ th argument of the set $Y=\left\{y_{1}, \ldots, y_{n}\right\}, \beta \in[0,1]$ and $\lambda$ and $\sigma$ are parameters such that $\lambda, \sigma \in(-\infty, \infty)-\{0\}$.

Note that the IMPOWAD operator accomplishes similar properties than the MPOWAD operator including monotonicity, idempotency, reflexivity, non-negativity 
and the boundary condition. It includes a wide range of particular cases by using different expressions in the weighting vectors and different values of $\lambda$ and $\sigma$. If $\lambda=\sigma=$ 1, the IMPOWAD operator becomes the IPOWAD operator as follows:

$$
\operatorname{IPOWAD}\left(\left\langle u_{1}, x_{1}, y_{1}\right\rangle, \ldots,\left\langle u_{n}, x_{n}, y_{n}\right\rangle\right)=\beta\left(\sum_{j=1}^{n} w_{j} b_{j}\right)+(1-\beta)\left(\sum_{i=1}^{n} v_{i}\left|x_{i}-y_{i}\right|\right),
$$

And if $\lambda=\sigma=2$, the IMPOWAD operator becomes the IEPOWAD operator:

$\operatorname{IEPOWAD}\left(\left\langle u_{1}, x_{1}, y_{1}\right\rangle, \ldots,\left\langle u_{n}, x_{n}, y_{n}\right\rangle\right)=\beta \sqrt{\left(\sum_{j=1}^{n} w_{j} b_{j}^{2}\right)}+(1-\beta) \sqrt{\left(\sum_{i=1}^{n} v_{i}\left|x_{i}-y_{i}\right|^{2}\right)}$.

\section{Reinsurance systems with OWA operators and distance measures}

\subsection{Suggested methodology}

From statistical techniques it is possible to evaluate different reinsurance programs, taking into account different criteria to make optimal decisions (maximum gain, minimum variance or probability of ruin), but with data themselves is not easy to choose a program. The experts must finally agree on a decision in accordance with the objectives of the company. This method uses fuzzy logic tools for evaluating the alternatives, through the following process: 
Step 1: Determine the characteristics or criteria that are most important in the decision. These criteria usually have a different numerical scale that can be unified using fuzzy numbers through membership functions on a scale of 0-1.

Step 2: From the results of the previous step a matrix $U=A x C$, containing the set of alternatives $A=\left\{A_{1}, A_{2}, \ldots A_{i}\right\}$ and the feature set $C=\left\{C_{1}, C_{2}, \ldots C_{j}\right\}$ is constructed.

Step 3: The optimal strategy where a group of experts defined the most appropriate value required for each feature on a scale of 0 to 1 is determined. The results will be aggregated using the vector $U=\left\{U_{1}, U_{2}, \ldots U_{j}\right\}$, such that $\sum_{J=1}^{n} U_{j}=1$ and $U_{j} \epsilon[0,1]$, which weights the view of each expert, unifying criteria of optimal characteristics for the vector $E=\left\{E_{1}, E_{2}, \ldots E_{j}\right\}$.

Step 4: The distances between the characteristics $C$ for each strategy $A$ and the vector of optimal strategy $E$ are calculated. The Minkowski distances will be the Hamming distance when $\lambda=1$ and the Euclidean distance when $\lambda=2$.

Step 5: The results of the previous step are added taking into account the weighted average with the vector $V=\left\{V_{1}, V_{2}, \ldots, V_{j}\right\}$, such that $\sum_{J=1}^{n} V_{j}=1$ and $V_{j} \in[0,1]$, the OWA with the vector $W=\left\{W_{1}, W_{2}, \ldots, W_{j}\right\}$, such that $\sum_{J=1}^{n} W_{j}=1$ and $W_{j} \in[0,1]$ and a vector of induction $I=\left\{I_{1}, I_{2}, \ldots, I_{j}\right\}$, which will be considered in the rankings and corresponds to the importance that the experts give to each one of the characteristics of the alternatives.

Step 6: The optimum alternative is chosen establishing a ranking of alternatives for every aggregation operators used in ascending order, since the shorter distance will be the best result. 


\subsection{Illustrative example}

The aim of this case based on the examples of Lemaire (1990) and De Andres and Terceño (2002), is to illustrate the decision-making process to choose the most suitable XL reinsurance program for a portfolio among different programs proposed by a reinsurance company. The criteria taken into account are:

- Criterion 1: Ratio: reinsurance premium / total premium transferor.

- Criterion 2: Coefficient of variation of the retained portfolio.

- Criterion 3: Probability of ruin.

- Criterion 4: Ratio: full / total premium transfer.

The suggested reinsurance programs are shown in the following Table 1:

Table 1: Characteristics of reinsurance programs

\begin{tabular}{ccccc}
\hline Programs & C1 & C2 & C3 & C4 \\
\hline XL1 & 0,040 & 3,85 & 0,000420 & 1,45 \\
XL2 & 0,030 & 4,07 & 0,000263 & 0,95 \\
XL3 & 0,035 & 4,36 & 0,000411 & 1,00 \\
XL4 & 0,042 & 3,92 & 0,000295 & 0,85 \\
XL5 & 0,028 & 3,80 & 0,000398 & 1,20 \\
XL6 & 0,025 & 4,50 & 0,000562 & 1,30 \\
\hline
\end{tabular}

As we can see at a glance whether the decision is made considering only one criterion, you may have other problems reflected in the other criteria, being necessary to 
have more tools to make the decision. To do this all data will be put in the same scale of 0-1 through fuzzy numbers with membership functions as well:

Criterion 1: Ratio: reinsurance premium / total premium transferor.

$$
U_{C_{1}}\left(x, C_{1}\right)=\left\{\begin{array}{lr}
1 & C_{1} \leq 0.025 \\
\left(\frac{0.05-C_{1}}{0.05-0.025}\right) & 0.025<C_{1}<0.05 \\
0 & C_{1} \geq 0.05
\end{array} .\right.
$$

Criterion 2: Coefficient of variation of the retained portfolio.

$$
U_{C_{2}}\left(x, C_{2}\right)=\left\{\begin{array}{lr}
1 & C_{2} \leq 3.8 \\
\left(\frac{5-C_{2}}{5-3.8}\right) & 3.8<C_{2}<5 \\
0 & C_{2} \geq 5
\end{array} .\right.
$$

Criterion 3: Probability of ruin.

$$
U_{C_{3}}\left(x, C_{3}\right)=\left\{\begin{array}{cc}
1 & 3 \leq 0.00028 \\
\left(\frac{0.0006-C_{3}}{0.0006-0.00028}\right) & 0.00028<C_{3}<0.0006 \\
0 & C_{3} \geq 0.0006
\end{array}\right.
$$

Criterion 4: Ratio: full / total premium transferor.

$$
U_{C_{4}}\left(x, C_{4}\right)= \begin{cases}0 & C_{4}=0 \\ C_{4} & C_{4} \leq 1 \\ 2-C_{4} & C_{4}>1 \\ 0 & C_{4} \geq 2\end{cases}
$$


From the above functions the following degrees of membership for each reinsurance program are obtained as shown in Table 2:

Table 2: Degrees of membership of criteria

\begin{tabular}{ccccc}
\hline Programs & C1 & C2 & C3 & C4 \\
\hline XL1 & 0,40 & 0,96 & 0,56 & 0,55 \\
XL2 & 0,80 & 0,78 & 1,00 & 0,95 \\
XL3 & 0,60 & 0,53 & 0,59 & 1,00 \\
XL4 & 0,32 & 0,90 & 0,95 & 0,85 \\
XL5 & 0,88 & 1,00 & 0,63 & 0,80 \\
XL6 & 1,00 & 0,42 & 0,12 & 0,70 \\
\hline
\end{tabular}

As noted, no program reaches the maximum level on all criteria and in contrast a good value in any of the criteria, is affected by a more negative effect on the others. Therefore, it has asked a group of experts to define the optimal level of each of the criteria, as shown in Table 3 below:

Table 3: Optimal level of criteria

\begin{tabular}{ccccc}
\hline Experts & C1 & C2 & C3 & C4 \\
\hline E1 & 0,9 & 0,8 & 0,8 & 0,9 \\
E2 & 0,8 & 0,7 & 0,9 & 0,8 \\
E3 & 0,9 & 0,8 & 0,7 & 0,8 \\
E4 & 0,9 & 0,7 & 0,8 & 0,9 \\
E5 & 0,9 & 0,9 & 0,7 & 0,8 \\
\hline
\end{tabular}


These expert opinions are aggregated with the arithmetic mean obtaining the following vector of optimal reinsurance presented in Table 4:

Table 4: Optimal reinsurance

\begin{tabular}{ccccc}
\hline Program & C1 & C2 & C3 & C4 \\
\hline$E$ & 0,88 & 0.78 & 0.78 & 0.84
\end{tabular}

The results have been added using the vectors $W=(0.35,0.25,0.20,0.20)$ and $V$ $=(0.2,0.3,0.2,0.3)$ and the induction vector $I=(0.87,0.77,0.80,0.84)$. Moreover, the expert opinions about the optimal reinsurance are aggregated with the vector $U=(0.3$, $0.3,0.2,0.2,0.1)$. The criteria for aggregations based on Minkowski distance will be the Hamming distance when $\lambda=1$ and the Euclidean distance when $\lambda=2$. The degree of importance of the weights (probabilities) is 0.6 while the OWA is 0.4 . The results are presented in Tables 5 and 6:

Table 5: Aggregated results 1

\begin{tabular}{ccccccccc}
\hline Program & NHD & NED & WHD & WED & OWAHD & OWAED & POWAD & EPOWAD \\
\hline XL1 & 0,29 & 0,31 & 0,28 & 0,30 & 0,32 & 0,34 & 0,296 & 0,316 \\
XL2 & 0,10 & 0,13 & 0,09 & 0,12 & 0,11 & 0,15 & 0,098 & 0,132 \\
XL3 & 0,22 & 0,22 & 0,22 & 0,22 & 0,23 & 0,23 & 0,224 & 0,224 \\
XL4 & 0,22 & 0,30 & 0,19 & 0,27 & 0,27 & 0,35 & 0,222 & 0,302 \\
XL5 & 0,10 & 0,13 & 0,11 & 0,14 & 0,12 & 0,15 & 0,114 & 0,144 \\
XL6 & 0,32 & 0,39 & 0,31 & 0,37 & 0,37 & 0,44 & 0,334 & 0,398 \\
\hline
\end{tabular}


Table 6: Aggregated results 2

\begin{tabular}{ccccccccc}
\hline Program & AOWAHD & AOWAED & IOWAHD & IOWAED & IAOWAHD & IAOWAED & IPOWAD & IEPOWAD \\
\hline XL1 & 0,27 & 0,29 & 0,32 & 0,34 & 0,27 & 0,29 & 0,296 & 0,316 \\
XL2 & 0,09 & 0,12 & 0,10 & 0,12 & 0,09 & 0,13 & 0,094 & 0,12 \\
XL3 & 0,21 & 0,21 & 0,23 & 0,23 & 0,22 & 0,23 & 0,224 & 0,224 \\
XL4 & 0,18 & 0,27 & 0,26 & 0,34 & 0,20 & 0,27 & 0,218 & 0,298 \\
XL5 & 0,08 & 0,12 & 0,08 & 0,12 & 0,12 & 0,15 & 0,098 & 0,132 \\
XL6 & 0,28 & 0,35 & 0,28 & 0,35 & 0,34 & 0,40 & 0,298 & 0,362 \\
\hline
\end{tabular}

Abbreviations: Normalized Hamming distance (NHD); Normalized Euclidean distance (NED); Weighted Hamming distance (WHD); Weighted Euclidean distance (WED); Ordered weighted averaging Hamming distance (OWAHD); Ordered weighted averaging Euclidean distance (OWAED); Probabilistic ordered weighted averaging distance (POWAD); Euclidean probabilistic ordered weighted averaging distance (EPOWAD); Ascending ordered weighted averaging Hamming distance (AOWAHD); Ascending ordered weighted averaging Euclidean distance (AOWAED); Induced ordered weighted averaging Hamming distance (IOWAHD); Induced ordered weighted averaging Euclidean distance (IOWAED); Induced ascending ordered weighted averaging Hamming distance (IAOWAHD); Induced ascending ordered weighted averaging Euclidean distance (IAOWAED); Induced probabilistic ordered weighted averaging distance (IPOWAD); Induced Euclidean probabilistic ordered weighted averaging distance (IEPOWAD).

Next, let us analyze the ordering of the alternatives with the results obtained shown in table 7: 
Table 7: Ranking of alternatives

\begin{tabular}{|c|c|}
\hline Method & Ranking \\
\hline NHD & $\mathrm{XL5}=\mathrm{XL2}<\mathrm{XL3}=\mathrm{XL4}<\mathrm{XL1}<\mathrm{XL6}$ \\
\hline NED & XL2 $=$ XL5 $<$ XL3 $<$ XL4 $<$ XL1 $<$ XL6 \\
\hline WHD & XL2 $<$ XL5 $<$ XL4 $<$ XL3 $<$ XL1 $<$ XL6 \\
\hline WED & XL2 $<$ XL5 $<$ XL3 $<$ XL4 $<$ XL1 $<$ XL6 \\
\hline OWAHD & $\mathrm{XL} 2<\mathrm{XL} 5<\mathrm{XL} 3<\mathrm{XL} 4<\mathrm{XL1}<\mathrm{XL6}$ \\
\hline OWAED & $\mathrm{XL2}=\mathrm{XL} 5<\mathrm{XL} 3<\mathrm{XL} 4<\mathrm{XL1}<\mathrm{XL6}$ \\
\hline POWAD & $\mathrm{XL} 2<\mathrm{XL} 5<\mathrm{XL} 4<\mathrm{XL} 3<\mathrm{XL1}<\mathrm{XL6}$ \\
\hline EPOWAD & XL2 $<$ XL5 $<$ XL3 $<$ XL4 $<$ XL1 $<$ XL6 \\
\hline AOWAHD & XL5 $<$ XL2 $<$ XL4 $<$ XL3 $<$ XL1 $<$ XL6 \\
\hline AOWAED & XL2 $=$ XL5 $<$ XL3 $<$ XL4 $<$ XL1 $<$ XL6 \\
\hline IOWAHD & XL5 $<$ XL2 $<$ XL3 $<$ XL4 $<$ XL6 $<$ XL1 \\
\hline IOWAED & $\mathrm{XL2}=\mathrm{XL5}<\mathrm{XL} 3<\mathrm{XL1}=\mathrm{XL} 4<\mathrm{XL6}$ \\
\hline IAOWAHD & XL2 $<$ XL5 $<$ XL4 $<$ XL3 $<$ XL1 $<$ XL6 \\
\hline IAOWAED & XL2 $<$ XL5 $<$ XL3 $<$ XL4 $<$ XL1 $<$ XL6 \\
\hline IPOWAD & $\mathrm{XL} 2<\mathrm{XL} 5<\mathrm{XL} 4<\mathrm{XL} 3<\mathrm{XL1}<\mathrm{XL6}$ \\
\hline IEPOWAD & XL2 $<$ XL5 $<$ XL3 $<$ XL4 $<$ XL1 $<$ XL6 \\
\hline
\end{tabular}

When ordering the distances of the alternatives from lowest to highest for each of the criteria it can be observed that XL2 is the best alternative when using the Hamming distance, WHD, OWAHD, IAOWAHD, POWAD and IPOWAD operator. AOWAHD and IOWAHD consider that XL5 is the best alternative, while NHD presents a tie between XL2 and XL5. Focusing on the Euclidean distance, NED, 
OWAED, AOWAED and IOWAED have a tie between XL2 and XL5, while WED, EPOWAD, IAOWAED and IEPOWAD indicate that XL2 is better.

\section{Conclusions}

This paper has presented a new method to evaluate reinsurance strategies using different tools such as fuzzy logic, membership functions, distance measures and OWA operators. With this method we have seen that very different criteria can be compared on the same scale to establish distances to the optimum level and then be added to enhance the decision-making process on reinsurance.

The Minkowski distance generalizes a wide range of distances such as the Hamming and Euclidean distance among others, taking into account multiple decision criteria for a more complete evaluation of the alternatives according to its characteristics. The great advantage of using the OWA operator is that it can consider a wide range of distances between the minimum and the maximum. Thus, the information is not lost in the process of analysis and the attitude of decision maker in the problem can be assessed. The main idea of this analysis is to consider a variety of scenarios from the most optimistic to the most pessimistic and select the method that best meets the goals of the company. Some key modern distance measures have been suggested for solving the reinsurance system including the IMOWAD and the IMPOWAD operator which include a wide range of particular cases.

Future research will consider other operators in the process of aggregation such as those that use inaccurate information in the analysis including intuitionistic fuzzy sets (He et al. 2014; Tao et al. 2014) and hesitant fuzzy sets (Zhao et al. 2014). The use of 
other distance measures and other applications will be also considered in the insurance field and other related disciplines.

\section{Acknowledgements}

Support from the MAPFRE Foundation and the European Commission through the project PIEF-GA-2011-300062, is gratefully acknowledged.

\section{References}

Beliakov, G., Pradera, A., \& Calvo, T. (2007). Aggregation functions: A guide for practitioners. Berlin: Springer-Verlag.

Belles-Sampera, J., Merigó, J. M., Guillén, M., \& Santolino, M. (2013). The connection between distorsion risk measures and ordered weighted averaging operators. Insurance: Mathematics and Economics, 52, 411-420.

Bolton J., Gader, P., \& Wilson, J. N. (2008). Discrete Choquet integral as a distance measure. IEEE Transactions on Fuzzy Systems, 16, 1107-1110.

De Andrés, J., \& Terceño Gómez, A. (2002). Actuarial applications of the theory of fuzzy subsets. CIMBAGE, 5, 1-39 (In Spanish).

Figueira, J., Greco, S., \& Ehrgott, M. (2005). Multiple criteria decision analysis: state of the art surveys. Boston: Springer.

Gil-Aluja, J. (1999). Elements for a theory of decision in uncertainty. Kluwer Academic Publishers, Dordrecht.

Grabisch, M., Marichal, J. L., Mesiar, R., \& Pap, E. (2011). Aggregation functions: Means. Information Sciences, 181, 1-22. 
Hamming, R. W. (1950). Error-detecting and error-correcting codes. Bell Systems Technical Journal, 29, 147-160.

He, Y. D., Chen, H. Y., Zhou, L. G., Liu, J. P., \& Tao, Z. F. (2014). Intuitionistic fuzzy geometric interaction averaging operators and their application to multi-criteria decision making. Information Sciences, 259, 142-159.

Kaufmann, A. (1975). Introduction to the theory of fuzzy subsets. Academic Press, New York.

Lemaire, J. (1990). Fuzzy Insurance. Astin Bulletin, 20, 33-55.

Merigó, J. M. (2013). The probabilistic weighted averaging distance and its application in group decision making. Kybernetes, 42, 686-697.

Merigó, J. M., \& Casanovas, M. (2011a). A new Minkowski distance based on induced aggregation operators. International Journal of Computational Intelligence Systems, 4, $123-133$.

Merigó, J. M., \& Casanovas, M. (2011b). Decision-making with distance measures and induced aggregation operators. Computers \& Industrial Enginering, 60, 66-76.

Merigó, J. M., \& Casanovas, M. (2011c). Induced aggregation operators in the Euclidean distance and its application in financial decision making. Expert Systems with Applications, 38, 7603-7608.

Merigó, J. M., \& Gil-Lafuente, A. M. (2010). New decision-making techniques and their application in the selection of financial products. Information Sciences, 180, 2085-2094.

Merigó, J. M., \& Gil-Lafuente, A. M. (2012). Decision-making techniques with similarity measures and OWA operators. SORT-Statistics and Operations Research Transactions, $36,81-101$.

Merigó, J. M., \& Gil-Lafuente, A. M. (2013). A method for decision making based on generalized aggregation operators. International Journal of Intelligent Systems, 28, 453473.

Merigó, J. M., Xu, Y. J., \& Zeng, S. Z. (2013). Group decision making with distance measures and probabilistic information. Knowledge-Based Systems, 40, 81-87. 
Merigó, J. M., \& Yager, R. R. (2013). Generalized moving averages, distance measures and OWA operators. International Journal of Uncertainty, Fuzziness and Knowledge-Based Systems, 21, 533-559.

Mondragón, J. R. (2009). Economic optimization of the ceded reinsurance: Decision models. Fundación Mapfre (In Spanish).

Su, W., Peng, W., Zeng, S., Peng, B., \& Pan, T. (2013). A method for fuzzy group decision making based on induced aggregation operators and Euclidean distance. International Transactions in Operational Research, 20, 579-594.

Tao, Z. F ., Chen, H. Y., Zhou, L. G., \& Liu, J. P. (2014). A generalized multiple attributes group decision making approach based on intuitionistic fuzzy sets. International Journal of Fuzzy Systems, 16, 184-195.

Xu, Z. S. (2012). Fuzzy ordered distance measures. Fuzzy Optimization and Decision Making, $11,73-97$.

Xu, Z. S., \& Chen, J. (2008). Ordered weighted distance measures. Journal of Systems Science and Systems Engineering, 17, 432-445.

Yager, R. R. (1988). On ordered weighted averaging aggregation operators in multicriteria decision-making. IEEE Transactions on Systems, Man and Cybernetics, 18, 183-190.

Yager, R. R. (1993). Families of OWA Operators. Fuzzy Sets and Systems, 59, 125-148.

Yager, R. R. (1998). Including importances in OWA aggregations using fuzzy systems modeling. IEEE Transactions on Fuzzy Systems, 6, 286-294.

Yager, R. R., \& Filev, D. P. (1999). Induced ordered weighted averaging operators. IEEE Transactions on Systems, Man and Cybernetics B, 29, 141-150.

Yager, R. R., Kacprzyk, J., \& Beliakov, G. (2011). Recent developments on the ordered weighted averaging operators: Theory and practice. Berlin: Springer-Verlag.

Zadeh, L. A. (1965). Fuzzy sets. Information and Control, 8, 338-353.

Zavadskas, E. K., \& Turskis, Z. (2011). Multiple criteria decision making (MCDM) methods in economics: An overview. Technological and Economic Development of Economy, 17, $397-427$. 
Zeng, S. Z. (2013a). Decision making with the uncertain IMOWAD operator. Kybernetes, 42, 785-799.

Zeng, S. Z. (2013b). Some intuitionistic fuzzy weighted distance measures and their application to group decision making. Group Decision and Negotiation, 22, 281-298.

Zhao, X. F., Lin, R., \& Wei, G. W. (2014). Hesitant triangular fuzzy information aggregation based on Einstein operations and their application to multiple attribute decision making. Expert Systems with Applications, 41, 1086-1094.

Zhou, L. G., Chen, H. Y., \& Liu, J. P. (2013). Continuous ordered weighted distance measure and its application to multiple attribute group decision making. Group Decision and Negotiation, 22, 739-758. 